\title{
Deep Learning Fundamentals
}

Katharina Breininger, Vincent Christlein, Tobias Würfl, Andreas Maier

Pattern Recognition Lab, Friedrich-Alexander-Universität, Erlangen, Germany katharina.breininger@fau.de

Deep learning has received a lot of attention in the machine learning community. Successful applications from speech recognition or computer vision are already part of our daily life. Much effort has been devoted to transferring this success to medical image computing. Therefore, neural networks have become an essential research direction. The first half of this tutorial is designed to familiarize participants with neural networks. The second half presents the transition from neural networks to deep learning.

The building blocks of classical neural networks, such as the multi-layer perceptron, activations and loss functions, are explained. Furthermore, the concepts of gradient-based learning and backpropagation to calculate the gradients are introduced.

The second part of the tutorial covers the elements of convolutional neural networks, around which most successful deep learning applications revolve. Special attention is devoted to regularization techniques, which are essential to stateof-the-art performance. Best practices and exemplary architectures conclude the tutorial. 